\title{
UTILIZATION OF ARTIFICIAL INTELLIGENCE FOR SENSITIVITY ANALYSIS IN THE STOCK MARKET
}

\author{
Zuzana Janková1 ${ }^{1}$ Petr Dostál ${ }^{1}$ \\ ${ }^{1}$ Institute of Informatics, Faculty of Business and Management, Brno University of Technology, Kolejní 2906/4, \\ Královo Pole, 61200 Brno, Czech Republic
}

To link to this article: https://doi.org/10.11118/actaun201967051269

Received: 14. 5. 2019, Accepted: 30. 9. 2019

To cite this article: JANKOVÁ ZUZANA, DOSTÁL PETR. 2019. Utilization of Artificial Intelligence for Sensitivity Analysis in the Stock Market. Acta Universitatis Agriculturae et Silviculturae Mendelianae Brunensis, 67(5): 12691283.

\begin{abstract}
The main contribution of this paper is to perform sensitivity analysis using artificial intelligence methods on the US stock market using alternative psychological indicators. The Takagi-Sugeno fuzzy model applies investor sentiment represented by VIX index and monitors the impact of economic optimism, political stability and control of the corruption index on the S\&P 500 stock index. Alternative psychological indicators have been chosen that have not been explored in the context of stock index performance sensitivity. Investors primarily use fundamental and technical analysis as a source to determine when and what to buy into an investment portfolio. However, psychological factors that may indicate the strength of reaction to the market are often neglected. Fuzzy rules are determined and tested using a neuro-fuzzy inference system and then the rules are reduced by fuzzy clustering to improve performance of ANFIS. The membership function is defined as a Gaussian function because it has the least RMSE value. The sensitivity analysis confirmed that there is a significant impact of the political stability index and the economic optimism index on the S\&P 500 performance. Conversely, the sensitivity analysis, unlike the previous study, did not confirm the strong impact of VIX on equity index performance. Results indicate that incorporating psychological indicators in macroeconomic models leads to better supervision and control of the financial markets.
\end{abstract}

Keywords: artificial intelligence, fuzzy approach, fuzzy logic, sensitivity analysis, sentiment, soft computing, stock market

\section{INTRODUCTION}

The financial industry has been increasingly dependent on advanced computing technology, especially to maintain its competitiveness in the global marketplace. The issue of capital market decision making using data mining techniques is one of the most important areas of finance. This has attracted a great deal of scientific interest and become a crucial research point providing for more accurate predictive and decision-making process. As reported by Tung and Le (2017), artificial intelligence resources such as fuzzy logic, artificial neural networks, and genetic algorithms are promising technologies with extensive application possibilities. More and more scientists and researchers have realized that the theory of fuzzy sets is a good tool to cope with uncertainties. Similarly, Dostál and Kruljacová (2018) add that fuzzy logic enables tolerance of inaccuracy or partial truth. As stated by Novák (2000), fuzzy logic makes it possible to cover inaccuracies and to work with the meanings of natural language words in a relatively simple way. The reason why fuzzy logic works is quite surprising because it uses vaguely characterized expert knowledge. 
It is the relationship between relevance and accuracy of information, that the principal of fuzzy logic Lotfi A. Zadeh (1965) called the principle of incompatibility. Fuzzy logic measures the uncertainty of the existence of phenomena using the notion of possibility. It measures the degree of presence/absence of a particular event on the scale. This degree is called the degree of membership in a set and is one of the fundamental elements of the theory of out of focus sets. The difference between Bayesian probability theory and fuzzy sets theory is in the different uses of the uncertainty type, i.e. stochastic versus linguistic. The difference is that the sum of the occurrence of a certain phenomenon does not have to correspond to a value of 1 , as assumed by classical probability theory. While classical set theory precisely defines membership and non-membership of elements in a set, a nonfuzzy set allows you to say about elements that more or less belong to the appropriate set of elements. Fuzzy logic works in the gray zone between yes and no, as Dostál (2011) notes. Wang and Wang (2015) report that fuzzy logic and neural networks have been increasingly used in the financial markets and widely recognized primarily for their ability to include nonlinear behaviour. The prices of investment instruments are influenced by deterministic, random factors and artificial intelligence methods, as reported by Chang et al. (2011), can overcome the problem of price uncertainty and non-linearity.

Dhaoui and Khraief (2014) used fuzzy logic to explain trading intensity and business trend changes. Empirical findings of sensitivity analysis show that pessimistic sentiment has a particularly significant impact on the development of the French financial market. Moreover, the results suggest that the impact of pessimism outweighs the impact of optimism on investment instrument yields. Analysis of sentiment is the result of increased use of the Internet and social media. Liu and Cocea (2017) report that sentiment analysis aims to recognize public attitudes or emotions through natural language processing, text analysis, and computer linguistics, suggesting the use of fuzzybased systems as computational models. Similarly, Jefferson et al. (2017) propose a system based on fuzzy rules for sentiment analysis, which offers more refined outputs using the fuzzy affiliation function. The authors state that the approach of fuzzy logic regarding language ambiguity is suitable for solving the given issue. As reported by Madhusudhan and Moorthi (2018), sentiment expresses what the public thinks and shares its positive or negative opinion. In general, opinions are unclear, and the acquisition of true sentiment poses a great challenge and requires effective methods of obtaining and summarizing them. The authors dealt with the analysis of sentiment using fuzzy logic. They concluded that fuzzy logic helps to increase the accuracy of the classification of opinions. Ghani et al. (2018) presented the principle of finding loyalty in decision-making based on fuzzy logic applied by a set of membership functions and a fuzzy rule system that classifies data in different types of loyalty. Implementing their approach provides high accuracy.

Babu and Kumar (2015) analyse the impact of the national sentiment and the investor sentiment on the stock market. Facebook GrossNational Happiness (FGNH) Index is considered the national sentiment and VIX is considered an indicator of the investor sentiment. A sensitivity analysis performed by neuro-fuzzy approach confirmed that there is a significant impact of the national income sentiment index and return of NSE. In addition, research has confirmed that a negative sentiment has a greater impact on return of the index than a positive sentiment. Kliger and Kudryavtsev (2013) focused on VIX. The aim of their study was to investigate the relationship between investors' market volatility expectations and their reaction to analyze stock returns. The authors found that positive (negative) excess return to recommendation upgrades (downgrades) are stronger when accompanied by decreases (increases) in the daily value of VIX. The authors argue that one possible explanation for this effect may be the fact that VIX can be seen as an indicator of future economic conditions. Namouri et al. (2017) investigate the relationship between investor sentiment and G7 stock market returns from June 1987 to February 2014. The investor sentiment, according to the authors, affects stock returns significantly and non-linearly, but its effects vary according to market conditions. Ruan (2018) analyzes the effect of market volatility on the stock market. Empirical results show that the VIX index has a significant impact on the equity market link. Sarwar (2014) examines cross-market differential relations of U.S. stock market uncertainty (VIX) with U.S. and European stock market returns before and during the European equity market crisis. The author found a strong negative relationship between VIX changes and European stock returns, which were twice as high in the stock market crisis as before.

Kliger and Levy (2003) took advantage of the option price data and found that the poor market mood is associated with the fact that investors are less, respectively more willing to tolerate risk. Kliger and Levy (2003) took advantage of the option price data and found that the poor market mood is associated with the fact that investors are less, respectively. more willing to tolerate risk. Similarly, Wright and Bower (1992) argue that people's mood affects their judgments due to uncertain future events by documenting that people in a good mood report a higher (lower) probability of positive future events and a lower (higher) probability of negative future events. Loewenstein et al. 
(2001) argues that VIX changes may negatively correlate with the current mood of investors. The basic intuition in this context is that good (bad) investors should perceive positive (negative) future financial results as more likely. Daszyńska-gygadło et al. (2014) tested the existence of a relationship between optimism index and returns at aggregate level in eight emerging markets. The authors use the sentiment and optimism of Thomson Reuters MarketPsych Indexes, which are based on scanning media coverage of relevant text reflecting individual moods and opinions. They found that there was a positive relationship between the investor's optimism index and the stock exchange returns surplus in Brazil and China. Excessive returns are further sensitive to changes in investor sentiment during the period of negative optimism in four of the eight markets surveyed.

Alam and Nadeem (2015) examine the impact of classical macroeconomic factors on the capital market in Pakistan, using regression analysis. They included one psychological factor, namely the dummy variable of political stability. Political stability has a positive relationship to the stock market. Their results indicate that the stock market index is rising during the period of dictatorship, while it is declining or showing instability during the democratic period. The stock market is negatively affected by government instability and uncertainty in the country, such as terrorist attacks, judicial crises and assassinations. In their study, Fernandes and Gonenc (2016) also use the index of political stability and the absence of violence in relation to international portfolio diversification. The authors concluded that political stability increases the trade-off between geographic and industrial diversification. Trabelsi (2017) deals with the influence of political insecurity caused by civil insurrection on the behavior and nature of the cycles of Tunisian stock markets over time. According to his research results, political uncertainty creates unstable financial markets and stronger stock market cycles. Perotti and Oijen (2001) are conducting a study in a number of emerging markets to determine if political shocks have any impact on stock markets. Their findings show drastic changes in excess returns when political risk increases or decreases, suggesting that political risk is an important pricing factor in the cross-section of equity returns. Similarly, Chau et al. (2014) examine the impact of political uncertainty on stock market volatility in MENA. Their outputs show a significant increase in the volatility of Islamic indices during periods of political unrest.

Aljazaerli et al. (2016) notes that the theoretical correlation between corruption and stock market developments is relatively extensive in the literature, but evidence of the impact of corruption on stock market developments remains contradictory and ambiguous. The authors examine the impact of corruption measured by the Corruption Perceptions Index on stock market developments. Based on a regression analysis, the authors confirm the positive impact of corruption on stock market developments. Omodero (2018) examines the impact of corruption using the Corruption Perception Index on Nigeria's stock market performance. The author used a multiregression analysis and found that there is a strong positive and significant relationship between input variables. Finally, the author recommends that the government formulate policies and rules that are more focused on combating corruption. Bolgorian (2011) focused on the role of stock market development on corruption. Its output suggests that there is a power-law dependency between corruption and stock market development. Furthermore, a negative relationship is observed between the level of corruption and the improvement of the financial system. Lau et al. (2013) assesses how corporate corruption affects equity market volatility. The authors have shown that the wider coverage of realized corporate corruption in the emerging markets is due to the decline in government policy uncertainty with regard to the business environment.

The aim of the paper is to conduct a sensitivity analysis on the US stock market using artificial intelligence, namely fuzzy logic, with the research being based and continue on previously conducted studies by Babu and Kumara (2015), Dhaoui and Khraief (2014). The neuro-fuzzy inference system and fuzzy clustering are used for sensitivity analysis. This research extends the previously published outputs on the combination of utilized artificial intelligence approaches. Specifically, the work uses adaptive neuro fuzzy inference system (ANFIS) and fuzzy clustering. Fuzzy clustering is chosen to reduce the rules and thus improve the performance of ANFIS. The work also expands the use of previously tested and new input variable, so far unexplored in the context of the impact of sensitivity analysis on stock index. Investors primarily use fundamental and technical analysis as a source to determine when and what to buy into an investment portfolio. However, psychological factors that may indicate the strength of reaction to the market are often neglected. In the end, the paper contributes to enriching the research dealing with stock market sensitivity analysis using artificial intelligence resources, which are not much published yet.

\section{MATERIALS AND METHODS}

\section{Methods}

Fuzzy inference system (FIS) consists of four main components: knowledge base, fuzzy inference system, fuzzification and defuzzification block. The described structure of a fuzzy inference system is 
graphically represented in the upper part of Fig. 1. The measured real variables are first transformed in a fuzzification block into linguistic variables. Two types of fuzzification are available. If the measured data are perfect, they are modelled as a crisp set. Noise data and stationary noise data are modelled as fuzzy sets. Dostál (2011) states that three to seven attributes of a variable are usually used. The degree of membership attributes of a given variable in a set is depicted by a mathematical function. There are several fuzzy membership functions: triangular, Gaussian, trapezoidal, sigmoidal, etc. Kayacan et al. (2018) add that the applications of the theory of fuzzy set membership functions are selected based on subjective perception of vague or imprecise categories. Furthermore, there are no criteria to assess the suitability or correctness of the selected membership function.

The fuzzification is followed by a fuzzy inference that defines the behaviour of the system using the IF-THEN rules and the language level evaluating the status of membership of the variable. Each combination of the attributes of the variables entering the system and occurring in the condition expresses one rule. Consequently, for each rule, the degree of support or the weight of the rule in the system needs to be determined. The result of fuzzy inference, as stated by Dostál et al. (2005), is a language variable. In most applications, however, the final output is required as a number, not a fuzzy set. As a result, the output fuzzy set must be converted to a number. Fuzzy rules define the connection between input and output fuzzy variables. Castillo et al. (2007) provide the following form of fuzzy rules used in Takagi-Sugeno (1985) fuzzy system:

$R^{n}$ : IF $X_{1}$ is $X_{1}^{n}$ and ... and $X_{l}$ is $X_{l}^{n}$ THEN $y$ is $Y^{n}$,

where $X_{l}^{n}$ is antecedent and $Y^{n}=\left[y^{n}, \bar{y}^{n}\right]$ is consequent. Here $y^{n}$ and $\bar{y}^{n}$, stated by Taskin and Kumbasar (2015), there may be consequences or linear functions:

$\underline{y}^{n}=\underline{a}_{1}^{n} x_{1}+\ldots+\underline{a}_{l}^{n} x_{l}+\underline{b}^{n}$,

$\bar{y}^{n}=\bar{a}_{1}^{n} x_{1}+\ldots+\bar{a}_{l}^{n} x_{l}+\bar{b}^{n}$,

wherein the antecedent of the fuzzy logic expression composed of one or more simple fuzzy terms associated with the fuzzy operators and the consequent is an expression that assigns the fuzzy values of the output variable. The inference system evaluates all rules and combines the weights of the consequent of all relevant rules into one fuzzy set using a summary operation.

The final step is defuzzification, which converts fuzzy inference results back to values. The purpose of this operation is to convert the fuzzy value of the output variable to best represent the results of the fuzzy calculation.
For stable FIS, according to Hammer et al. (2012), it is necessary to choose the appropriate system parameters and structure. These must be set or derived using the learning process. Enough input and output variables are a prerequisite. The most commonly used system for determining the fuzzy inference system rules of the Takagi-Sugeno type is the adaptive neuro-fuzzy inference system (ANFIS). ANFIS makes it possible to combine human thinking of fuzzy systems with the neural network training and interconnection structure.

The ANFIS structure is made up of 5 layers, as shown in the lower part of Fig. 1. Zitta and Palatová (2005) describe the ANFIS structure as follows: Layer 1 consists of adaptive nodes with transfer functions that correspond to the functions of the linguistic values. In other words, each node represents the linguistic value of the input variable. Layer 2 consists of non-adaptive nodes that multiply the incoming signals and output the weight of the rules. Layer 3 is also made up of non-adaptive nodes that output the weight ratio of each rule to the sum of the weights of all rules. Layer 4 is formed by adaptive nodes with a transfer function corresponding to the desired shape of the consequence. The last 5 layers are one non-adaptive node that calculates the total output as the sum of all input signals.

The number of fuzzy rules created by ANFIS is subsequently reduced by the fuzzy clustering method. In the case of fuzzy clustering, each element is assigned a membership level, and in the case of soft clustering, the element can belong to not only one cluster. The membership level indicates the strength of the assignment between the element and the corresponding cluster. Fuzzy clustering, as Dostál (2011) describes, is the process of assigning these membership levels. Fuzzy C-Means (FCM) is one of the most common fuzzy clustering algorithms. The FCM algorithm creates a distribution of a finite set of $n$ elements $X=\left\{x_{1}, X_{2}, \ldots, x_{n}\right\}$ into the file fuzzy clusters with respect to the given criterion. To determine the finite number of elements, the algorithm returns a list of $c$ centroids $C=\left\{C_{1}, C_{2}, \ldots, C_{n}\right\}$ and matrix $W=w_{i j} \in[0,1], i=1,2, \ldots, \mathrm{n}, j=1,2, \ldots, c$, where each element $w_{i, j}$ denotes the degree of membership as the element $X_{i}$ belongs to the cluster $c_{i}$. The aim of the FCM method is to minimize the purpose function:

$$
w_{k}(x)=\frac{1}{\sum_{j} \frac{d\left(\text { center }_{k x}\right)^{2 /(m-1)}}{d\left(\text { center }_{j x}\right)}} .
$$

The fuzzifier $m$ determines the degree of cluster fuzzification. In case the limits of $m=1$, membership $w_{i, j}$ converges to 0 or 1 , leading to a crisp distribution. In case of lack of experiments or knowledge, $m$ is chosen to be equal to 2 . The result 


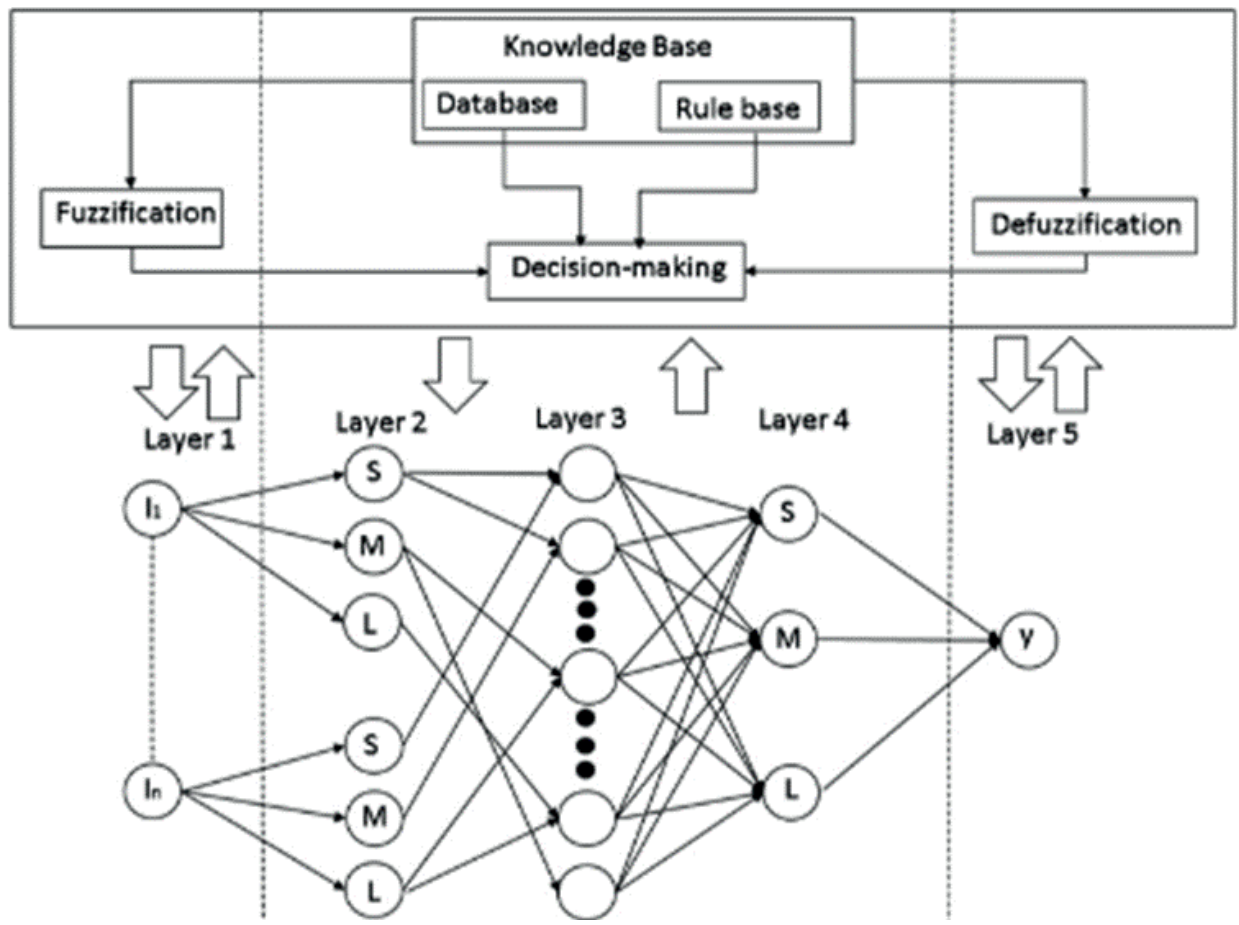

1: ANFIS structure

Source: Garcia et al. (2018)

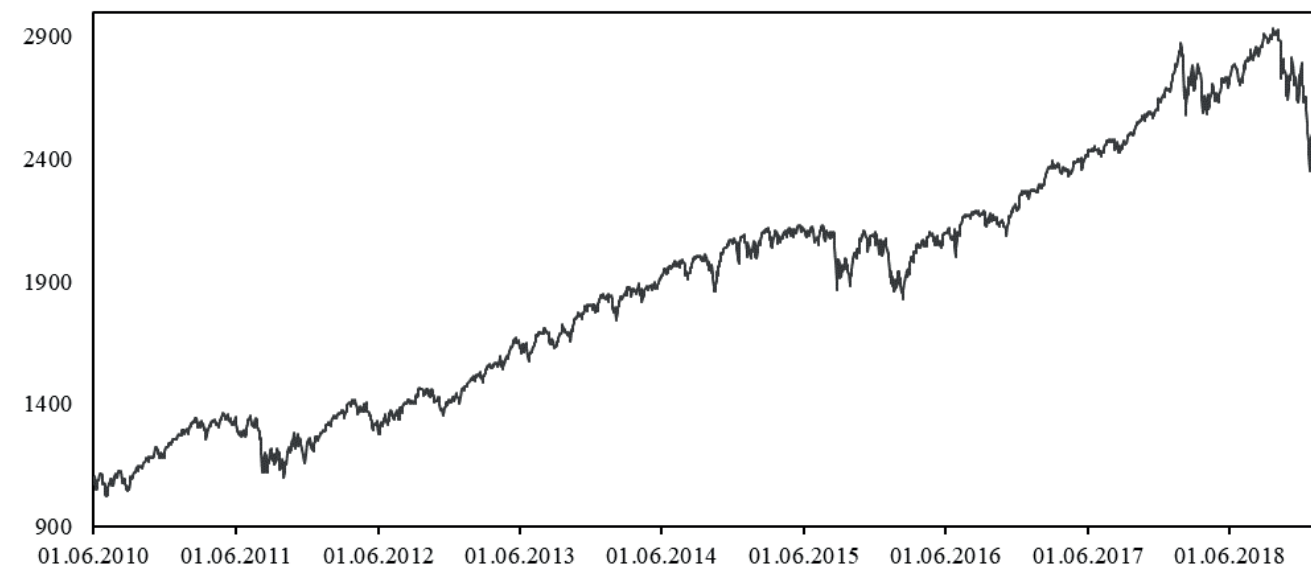

2: Historical development of the S\&P 500 index

of the FCM algorithm is $n$ data about elements $\left(x_{1}, X_{2}, \ldots, X_{n}\right)$ that are clustered, the number of $c$ clusters with centroid coordinates $\left(c_{1}, c_{2}, \ldots, c_{n}\right)$ and $m$ as a degree of cluster fuzzification. For fuzzy c-means, the centroid of the cluster is calculated as the average of all points, weighted by its degree of belonging to the cluster according to the formula:

$c_{k}=\frac{\sum_{x} w_{k}(x) x}{\sum_{x} w_{k}(x)}$

Each point $x$ is determined by a set of coefficients, which indicates the degree of membership of the $k$-th cluster $w_{k}(x)$. The degree of membership $w_{k}(x)$ is related inversely with the distance $x$ from the centroid. It also depends on the parameter that affects how much weight the closest centroid belongs to.

\section{Materials}

The research examines the relationship between the investor's national sentiment represented by the VIX index, economic optimism, the political stability index and the control of corruption index against the Standard and Poor's 500 index on the US stock market. The S\&P 500 index and other indices are collected from the Yahoo Finance (2019) and World Bank Finance websites (2019). The data sample is selected for the period from 2010 to 2018. 
Fig. 2 shows the historical development of the S\&P 500 index.

The S\&P 500 is based on market capitalization of the 500 largest companies that have stock listed on the NYSE and NASDAQ. Since the stock index value is high and the S\&P 500 index differences are quite significant, which can negatively affect the performance of the network, it is necessary to pre-process the original data using the following Boyacioglu and Avci (2010) procedure:

$r_{t}=\ln \left(\frac{y_{t}}{y_{t-1}}\right)$,

wherein $r_{t}$ represents the return of the S\&P 500 at time $t, y_{t}$ and $y_{t-1}$ are the monthly index values at time $t$, and time $t-1$, respectively.

The implied volatility index (VIX) is chosen to capture the expected market volatility of investors launched by the Chicago Board Options Exchange (CBOE) in 1993. VIX is based on the S\&P 500 index options. The index is calculated in real time and is continuously published during each business day. VIX can also be seen as an indicator of future economic conditions and is known as the "fear gauge". Overall, the index of political stability is important for understanding the role of political certainty or uncertainty in stock market stability and is of great importance to market investors and regulators. The destabilization of the political scene, according to the above research, causes stock market volatility. The theoretical correlation between corruption and stock market developments is widely discussed in the literature, but evidence of the impact of corruption on stock market developments remains contradictory and ambiguous. For this reason, the psychological index is incorporated into the model and its influence on the US stock market is monitored. The last input variable is index of economic optimism. The sensitivity of investor moods to the main US stock index returns is being observed, as positive mood investors tend to make optimistic judgments and ignore investment-related risks, while people with a negative mood tend to make pessimistic judgments and are not willing to tolerate the risk associated with investment.

Tab. I shows an example of the source data entering the created model for each reference year. The value of the political stability index ranges from -2.5 (weak political scene stability) to 2.5 (strong political scene stability). For the US, the index value for the reporting period is from 0.3 to 0.68 . The values of control of corruption index are set in the range of -2.5 (weak corruption control) to 2.5 (strong corruption control) as well as the political stability index. The index value in the US ranged from 1.27 to 1.41 in the selected period. VIX is also known by other names like "Fear Gauge" or "Fear Index." Investors, research analysts and portfolio managers look to VIX values as a way to measure market risk, fear and stress before they take investment decisions. A higher values of index of economic optimism should be taken as positive/ bullish, while a lower values of this index should be taken as negative/bearish. Data and their values are listed and discussed below, especially in the chapter Results.

Tab. II summarizes statistic parameters for inputs and output. On average, the adjusted return on the S\&P 500 index for the reporting period was 0.010 . Other average values of input quantities are listed in the table. S\&P 500, control of corruption index and VIX are very centralized nearly to its average level. Index of political stability and index of economic optimism are centralized on average nearly its maximal level.

Tab. III shows the correlation coefficients of selected input variables and output variable. Also,

I: Annual source data

\begin{tabular}{lrrrrrrrr}
\hline \multicolumn{1}{c}{ Year } & 2010 & 2011 & 2012 & 2013 & 2014 & 2015 & 2016 & 2017 \\
\hline Index of political stability & 0.44 & 0.59 & 0.63 & 0.64 & 0.58 & 0.68 & 0.40 & 0.30 \\
Control of corruption index & 1.27 & 1.27 & 1.41 & 1.31 & 1.38 & 1.40 & 1.37 & 1.38 \\
VIX & 17.75 & 23.40 & 18.02 & 13.72 & 19.20 & 18.21 & 14.04 & 11.04 \\
Index of economic optimism & 45.80 & 42.80 & 45.10 & 43.10 & 48.40 & 47.20 & 54.80 & 51.90 \\
\hline
\end{tabular}

II: Summary statistic for inputs and output variables

\begin{tabular}{lcccccc}
\hline \multicolumn{1}{c}{ Statistics } & Mean & Max & Min & Sd & Skewness & Kurtois \\
\hline Adjusted return of S\&P 500 & 0.010 & 0.102 & -0.074 & 0.033 & -0.142 & 0.590 \\
Index of political stability & 0.538 & 0.680 & 0.300 & 0.128 & -0.736 & -0.939 \\
Control of corruption index & 1.353 & 1.410 & 1.270 & 0.051 & -0.651 & -1.184 \\
VIX & 17.015 & 42.960 & 9.510 & 5.702 & 1.810 & 4.597 \\
Index of economic optimism & 47.192 & 56.400 & 35.800 & 3.873 & -0.058 & 0.350 \\
\hline
\end{tabular}


III: Matrix of Correlation Coefficients

\begin{tabular}{lccccc}
\hline \multicolumn{1}{c}{ Correlation Matrix } & $\begin{array}{c}\text { Adjusted return } \\
\text { of S\&P 500 }\end{array}$ & $\begin{array}{c}\text { Index of political } \\
\text { stability }\end{array}$ & $\begin{array}{c}\text { Control of } \\
\text { corruption index }\end{array}$ & VIX & $\begin{array}{c}\text { Index of economic } \\
\text { optimism }\end{array}$ \\
\hline Adjusted return of S\&P 500 & 1 & $0.1704^{*}$ & 0.04603 & $-0.4245^{*}$ & $0.1546^{*}$ \\
Index of political stability & $0.1704^{*}$ & 1 & 0.0065 & $-0.2569^{*}$ & $0.4616^{*}$ \\
Control of corruption index & 0.0460 & 0.0065 & 1 & $-0.4417^{*}$ & $0.5199^{*}$ \\
VIX & $-0.4245^{*}$ & $-0.2569^{*}$ & $-0.4417^{*}$ & 1 & $-0.4794^{*}$ \\
Index of economic optimism & $0.1546^{*}$ & $0.4616^{*}$ & $0.5199^{*}$ & $-0.4794^{*}$ & 1 \\
\hline
\end{tabular}

* correlation coefficients are statistically significant at the confidence interval of 90\%

statistical significance at the confidence interval of $90 \%$ is shown. It is clear from the table that the only input variable is negatively correlated with the adjusted return of the examined stock index. It is the input variable VIX with a correlation value of -0.4245 . Other input variables show a positive correlation with the adjusted return of S\&P 500. The strong positive dependence shows the index of economic optimism with a correlation coefficient of 0.1546 also index of political stability with a correlation coefficient of 0.1704. A control of corruption index shows a very low dependence with the S\&P 500 index of 0.0460 can be designated after an independent variable.

\section{RESULTS}

The created fuzzy inference system is shown in Fig. 3. Four variables enter the FIS: the index of political stability, the control of corruption index, the VIX and the index of economic optimism. The output of the model is the adjusted return of the S\&P 500 stock index. It is used the FIS type TakagiSugeno, which unlike Mamadani type, can work with the system ANFIS.

Three attributes of the basic variables (LOW, MEDIUM, HIGH) are used mainly due to the distribution of the data file and recommendations of experts in the field of fuzzy logic such as Dostál (2011). The degree of the membership attribute of a given variable in a fuzzy set is represented by a mathematical function. Due to the nature of the data used corresponding to the most Gaussian distribution, this type of membership function is chosen. Similarly, the Gaussian membership function for testing data from financial markets is recommended by the authors Esfahanipoura and Aghamiri (2010), who used this feature in their ANFIS model to test investment instrument data in their research. Furthermore, the Gaussian function is also recommended by Talpur et al. (2017).

The fuzzy membership function for the political stability index is shown in Fig 4. The political stability index measures the perception that the country's government is stable and will not be destabilized or overthrown by unconstitutional or violent means. The value of the political stability index ranges from -2.5 (weak political scene stability) to 2.5 (strong political scene stability). For the US, the index value for the reporting period is from 0.3 to 0.68 .

Fig. 5 shows a fuzzy membership function for control of the corruption index that captures the perception of the extent to which public authority is exercised for private profit, including various forms of corruption, as well as the capture of the power of the state elites for private purposes and interests. Again, the values of this index are set in the range of -2.5 (weak corruption control) to 2.5 (strong corruption control) as well as the political stability index. The index value in the US ranged from 1.27 to 1.41 in the selected period.

Fig. 6 illustrates the VIX volatility index membership function. The VIX Index or Fear

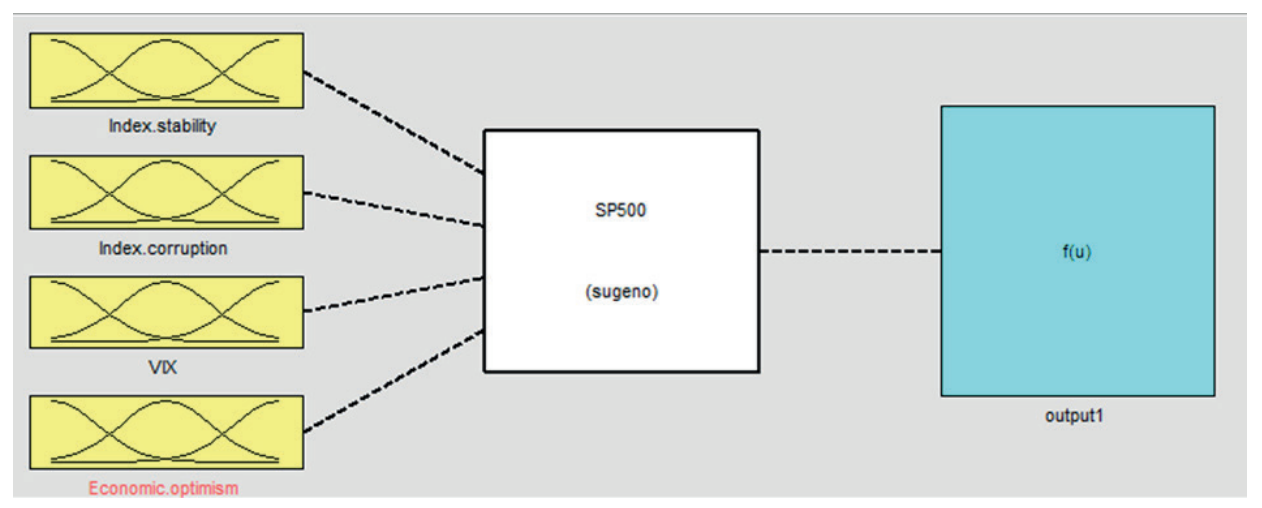

3: Fuzzy inference system 


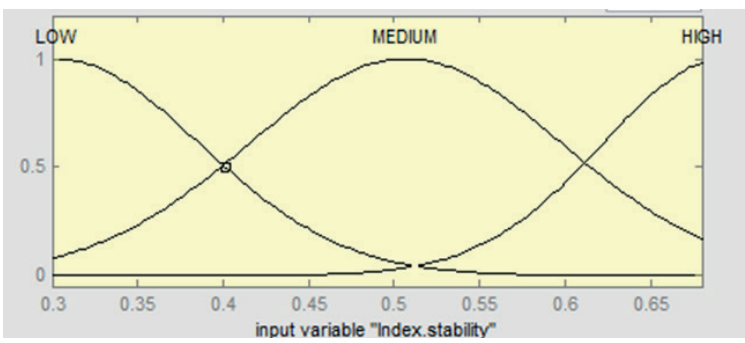

4: Fig 4: Input variable of political stability index

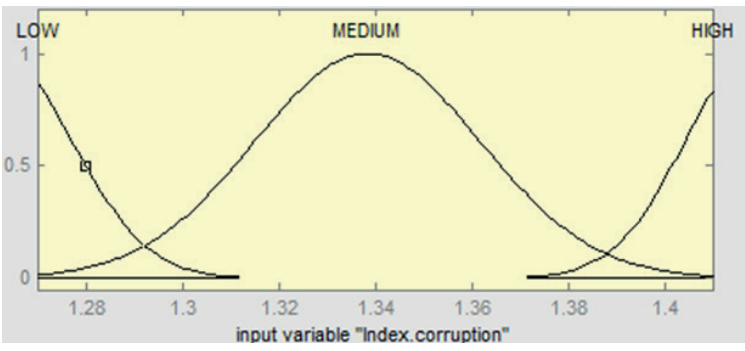

5: Fig 5: Input variable of control of corruption index

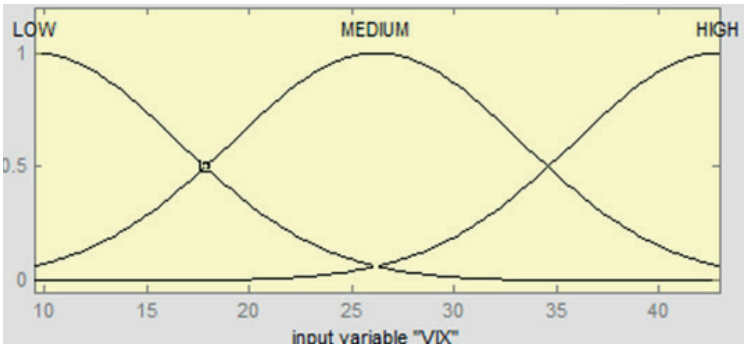

6: Fig 6: Input variable of VIX

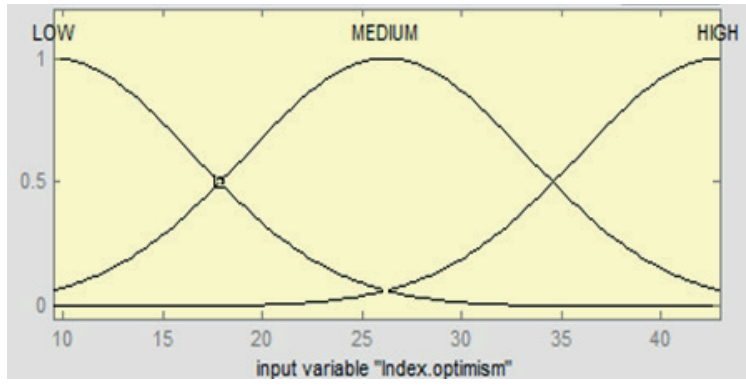

7: Fig 7: Input variable of economic optimism index

Index is a measure of stock market volatility. The index value is derived from the volatility of option contracts to the S\&P 500. The index basically records volatility and changes in prices of certain investment instruments. If the volatility is large, the price of investment instruments moves rapidly and significantly, increasing the VIX index and the risks of the instrument. VIX ranged from 9.51 to 42.96 in the reporting period.

The membership function for the index of economic optimism is shown in Fig. 7. The economic optimism index assesses the relative level of economic conditions in the US, including the six-month economic outlook, personal financial perspective and confidence in federal economic policies. The level of 50 is referred to as economic optimism. Values below 50 are referred to as economic pessimism. In the US, the index for that period ranged from 35.8 to 58 .

In the proposed 4-input and 1-output fuzzy model, the IF-THEN rules are determined using ANFIS. ANFIS is a forward neural network that uses a hybrid learning method for network learning. Parameters in the antecedent rule are optimized by the gradient method, the parameters of the consequence are calculated by the least squares method. For this model, the number of training epochs is 10 and training error tolerance is set to zero. The training process stops whenever the maximum epoch number is reached or the training error goal is achieved. For splitting the data into training and testing purpose, according to literature (Salleh et al., 2018; Shrivastava and Srid-haran, 2013) most researchers practiced the $70 \%$ training and 30\% testing because the more data applied for the training, the more optimal and accurate results a system generates. Therefore, in this study the $70 \%$ of the dataset instances were selected for training set and the remaining $30 \%$ of the dataset instances were chosen for testing set. The first 70\% of the dataset is used for training and the last 30\% of the dataset for testing. ANFIS generated a total of 81 rules. Fig. 8 shows ANFIS structure for model. The total ANFIS model errors are 0.0163 and ANFIS training completed at epoch 2. Examples of rules created using ANFIS are as follows:

IF (index political stability is LOW) and (control of corruption index is LOW) and (VIX is HIGH)

and (index of economic optimism is LOW) THEN (return of S\&P 500 is LOW)

$O R$

IF (index political stability is MEDIUM) and (control of corruption index is MEDIUM) and (VIX is MEDIUM)

and (index of economic optimism is MEDIUM) THEN (return of S\&P 500 is MEDIUM)

$O R$

IF (index political stability is HIGH) and (control of corruption index is HIGH) and (VIX is LOW)

and (index of economic optimism is HIGH) THEN (return of S\&P 500 is HIGH).

Subsequently, the number of rules is reduced by c-mean fuzzy clustering according to the procedure outlined in Materials and Methods. Fig. 9 shows the resulting rule block after clustering. 


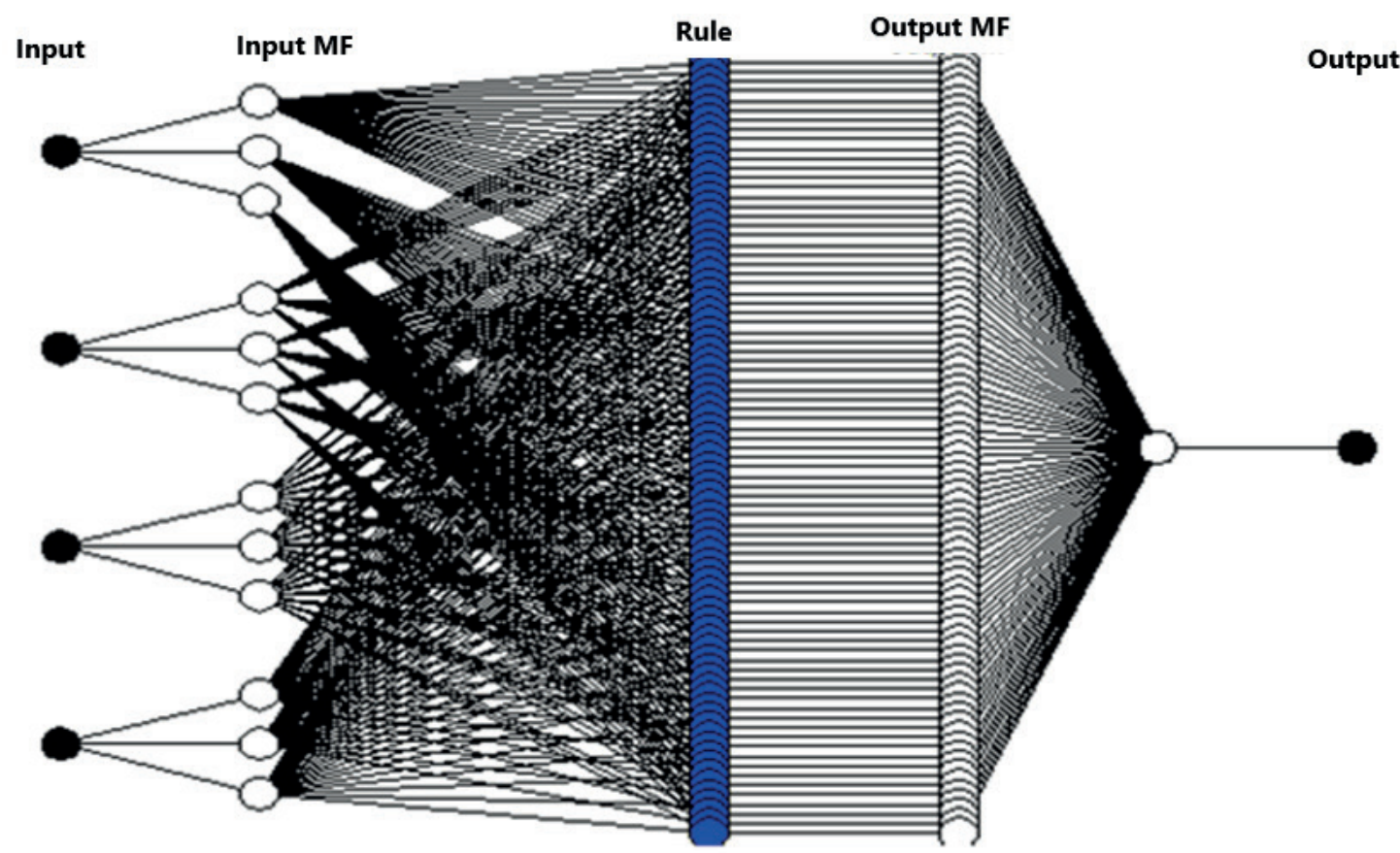

8: ANFIS structure for model

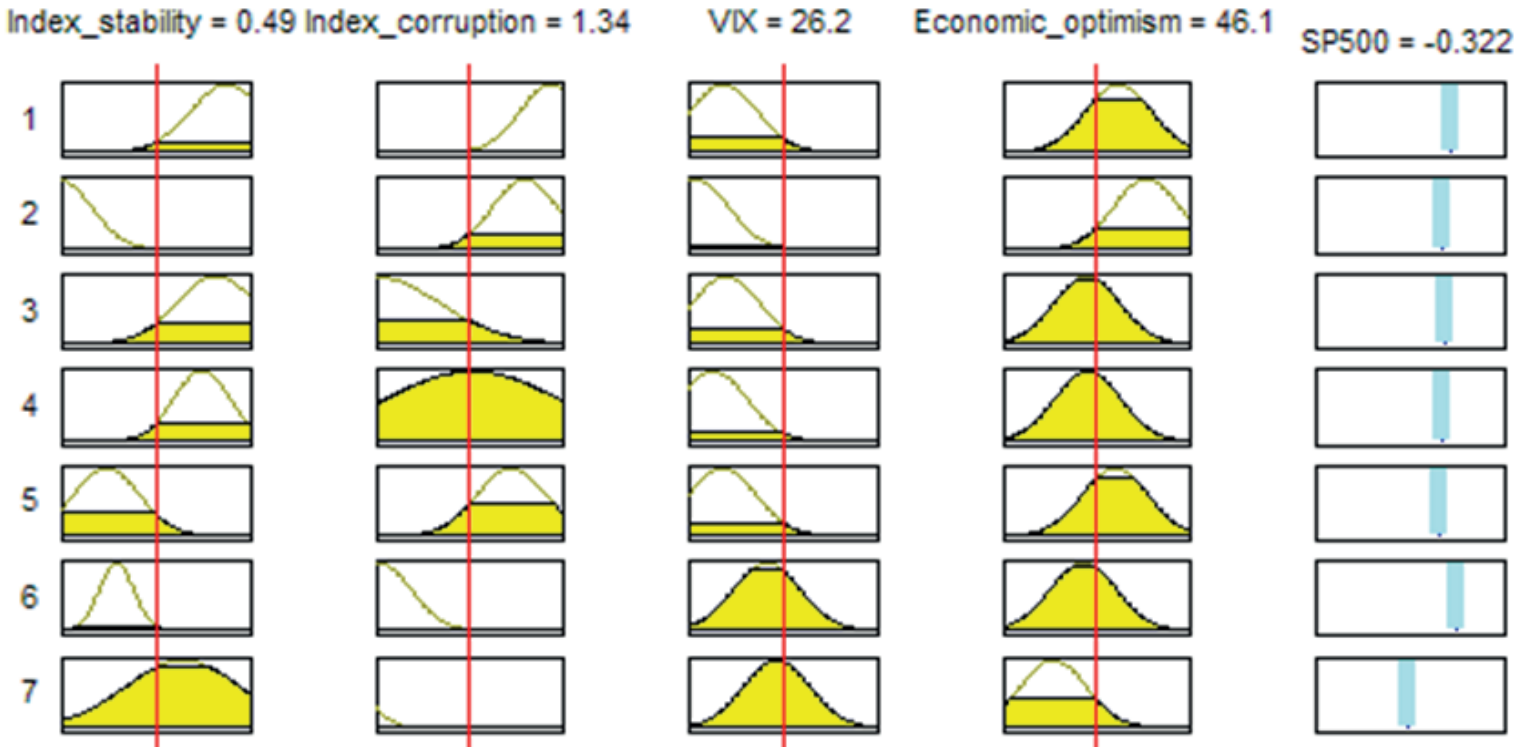

9: The resulting rule block after clustering

Fuzzy clustering created a total of seven clusters of fuzzy rules. The rules based on clustering are listed by line. The input variables are represented in the four columns. The last column marks the output variable obtained from the created model. The resulting block of rules can also be used to evaluate the model situation. According to the set values of the input variables, it can be stated that the political stability index is 0.49 , the corruption control index 1.34, VIX 26.2 and economic optimism 46.1. According to the fuzzy model, the adjusted S\&P $500-0.322$ index return is achieved. This does not apply only to one input variable in isolation, but the model is created for four input variables, so the investment decision is based on all inputs.

Fig. 10 to 12 represent a sensitivity analysis obtained by fuzzy logic. Fig. 10 shows the sensitivity of the political stability index and VIX to the adjusted return of the S\&P 500 index. If the US political stability index is low, it can be seen that the S\&P 500 stock index is also low to negative, and assets can be regarded as "SELL". Investors in an unstable political environment are worried about the consequences of governments and sell their investment instruments. If the government is stable and there is no threat of destabilization or its 


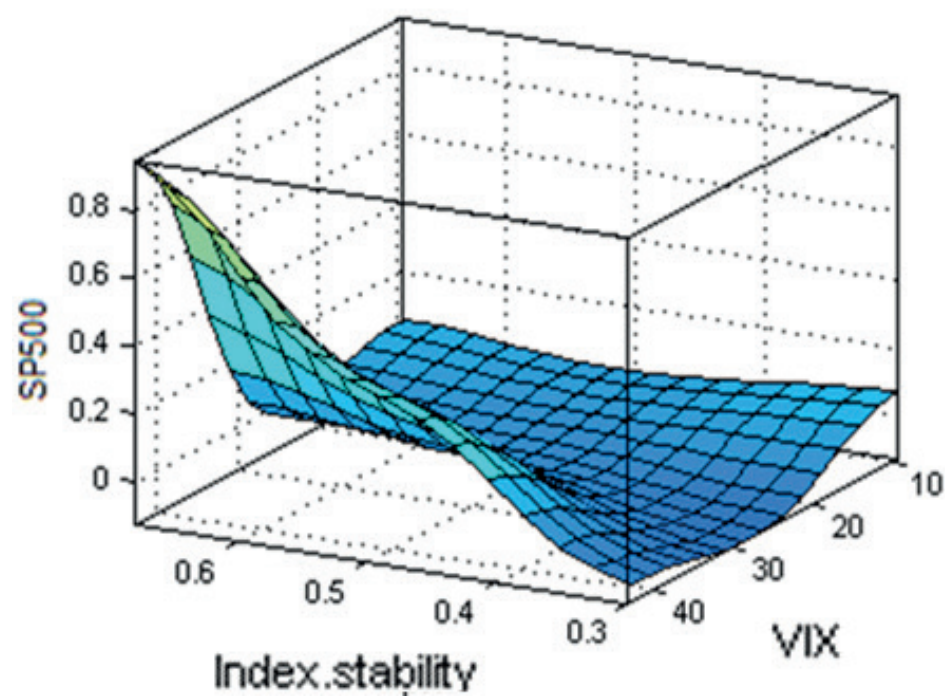

10: Sensitivity analysis of the political stability index and VIX

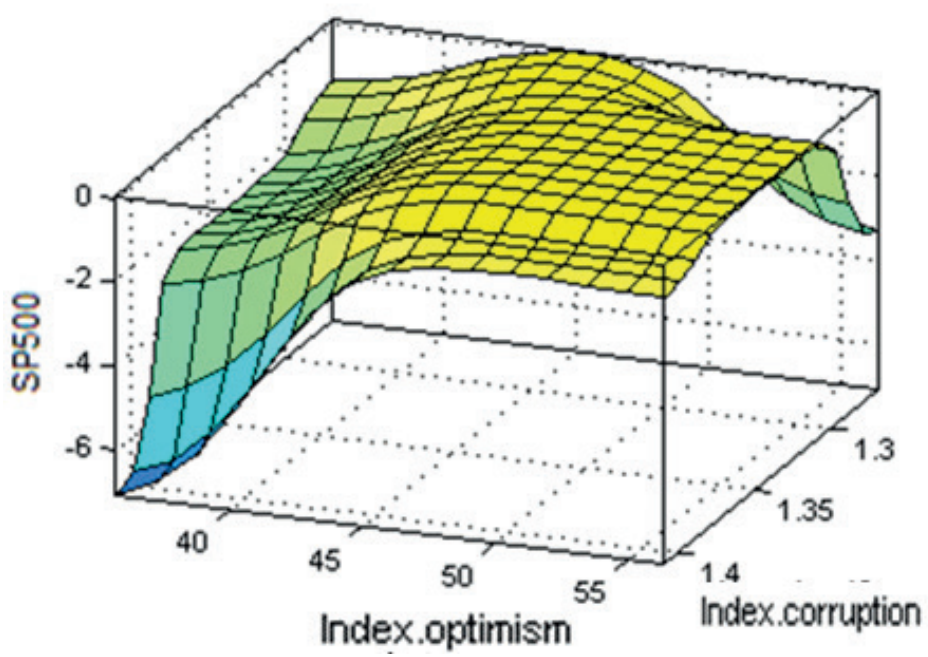

11: Sensitivity analysis of the economic optimism index and control of corruption index

overthrow, the political stability index is medium or high. In this case, a strong influence on the adjusted return of the S\&P 500 index can be observed. Changing the range of the political stability input variables causes an overall significant and aggressive change in the market trend.

The same picture also shows the sensitivity of the fear index to the value of the S\&P 500 index. In the case of low volatility of the VIX index, the market trend is growing. Conversely, in the event of a downward trend in the market, the volatility of stock instruments increases and investor fear and the risk of buying instruments into the portfolio are growing. Moreover, the change in range for the VIX variable is insignificant on the adjusted return of the S\&P 500 index output variable. According to the results, the influence of the VIX index is not as fundamental as the influence of the political stability index. It can be observed that the weight of this two inputs is not identical in terms of impact on individual output. The effect is not symmetric.

Fig. 11 shows the sensitivity of the economic optimism index and the control of the corruption index to the adjusted return of the S\&P 500 index. When the control of corruption index is low, the market trend is labelled as falling, or the stock index return is significantly reduced even by a slight change in the index. On the other hand, high control and anti-corruption in the country increase the return of the S\&P 500 stock index and investors are starting to buy stock titles as the trade trend is described as growing. Despite of this, the figure shows that the sensitivity of the value of the S\&P 500 index to the change in rage of the control of the corruption index remains about the same with a non-significant difference in sensitivity when 


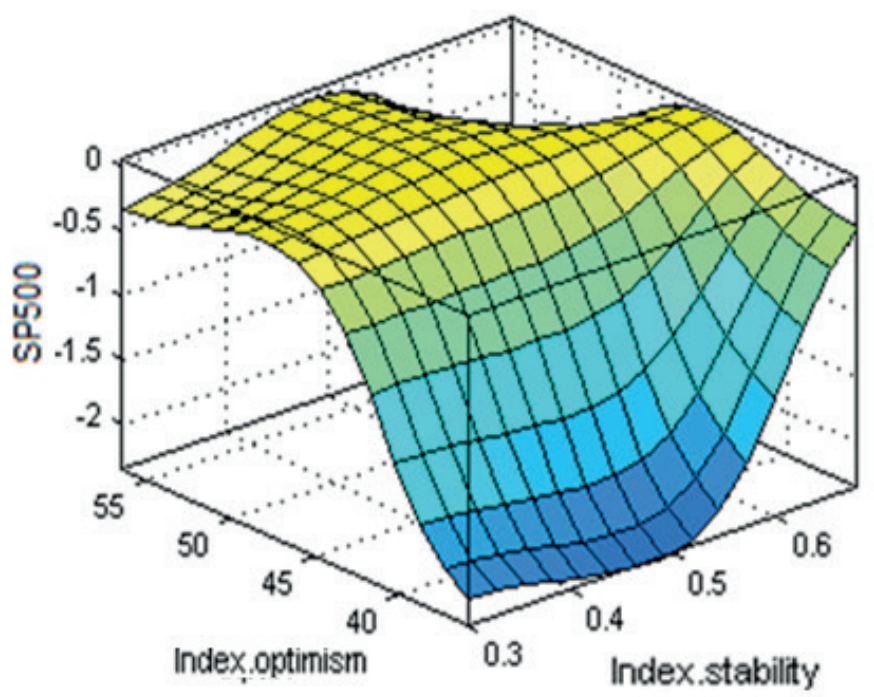

12: Sensitivity analysis of economic optimism and political stability index

the output variables take the level of null return. It can be noted that economic optimism has a greater influence on the value of the equity index return than the corruption index. Economic optimism is further discussed below.

Fig. 12 depicts the sensitivity of economic optimism to the stock index return on the the adjusted return of the S\&P 500 index. The S\&P 500 index is growing at a strong pace when the country's economic pessimism and economic optimism are changing. When economic optimism is achieved, as shown by the index of 50, the business trend slows down, and the stock index growth stagnates. When the stock index achieves losses, it can be seen from the graphs that investors reduce their business activity dramatically, which corresponds to high pessimism and low optimism. It can be clearly seen that the change in range for the optimism affects significantly the market trend. When economic optimism is "Medium" or "High" return of S\&P 500 index become "Increase" and "Buy". However "Low" range of economic optimism cause dramatically "Decrease". Overall, the change in range for the optimism input variable induces significant a change in the market trend.

Finally, the appropriateness of the membership function used is verified. Gaussian membership functions are compared with a triangular, trapezoidal and bell membership functions. The indicators RMS, $\mathrm{R}^{2}$ and MAPE are chosen to determine the suitability of the function. The predictive ability of the created model is determined by comparing the original data and outputs obtained from the model. For this purpose, the root-mean-square error (RMSE) pointer is used, the mathematical notation of which is as follows:

$R M S E=\sqrt{\frac{1}{n} \sum_{t=1}^{n}\left(y_{t}-\dot{y}_{t}\right)^{2}}$.
The predictive ability of the created model is further evaluated using the determination coefficient $\left(\mathrm{R}^{2}\right)$ calculated according to the following mathematical formula:

$R^{2}=1-\sum_{t=1}^{n} \frac{\left(y_{t}-\dot{y}_{t}\right)^{2}}{\dot{y}_{t}^{2}}$

In addition to the above statistical parameters can be used to evaluate the performance of the model, mean absolute percentage error (MAPE) with mathematical notation:

$M A P E=\frac{1}{n} \sum_{t=1}^{n} \frac{\left|y_{t}-\dot{y}_{t}\right|}{y_{t}} \times 100$,

where $n$ is number of observations of the data set, $y_{t}$ indicates the output predicted by the model for t-th value, $\dot{y}_{t}$ indicates the original measured output of data set for t-th value.

Results of comparison of membership functions are set forth in Tab. IV. The accuracy of the generated model is improved using a suitable membership function, which is indicated by lower values of RMS, $\mathrm{R}^{2}$ and MAPE. Membership functions must correspond to the character of the data used in the model. The table shows that the algorithm obtained using gaussian and bell functions provides the most optimal ANFIS topology. The topology of the Gaussian membership function gained RMS 0.06134 value and MAPE 5.11\%. A very similar error was provided by the model created by the bell membership function with RMS 0.01665 value and MAPE 5.83\%. These values are promising. As a result of the function testing, it can be stated that the appropriate function was used for this sensitivity analysis on the American stock market and the distribution pattern of the data set used best corresponds to the Gaussian distribution. 
IV: Comparison of membership functions

\begin{tabular}{lccc}
\hline Used membership function & $\mathrm{RMS}$ & $\mathrm{R}^{2}$ & MAPE \\
\hline Triangular & 0.01761 & 0.55 & 8.75 \\
Trapezoidal & 0.02262 & 0.49 & 7.21 \\
Bell & 0.01665 & 0.56 & 5.83 \\
Gaussian & 0.01634 & 0.85 & 5.11 \\
\hline
\end{tabular}

V: Statistical analysis of output using MLR and ANFIS

\begin{tabular}{lcc}
\hline Used approach & $\mathrm{R}^{2}$ & RMSE \\
\hline MLR & 0,26 & 0,02913 \\
ANFIS & 0.85 & 0.01634 \\
\hline
\end{tabular}

Furthermore, the generated neuro-fuzzy model is compared with multidimensional regression analysis. If $X_{t}$ corresponds to the process parameters and $\varepsilon_{t}$ indicates a predictive error, then the regression analysis entry is as follows:

$Y_{t}=b_{0}+b_{1} X_{t 1}+b_{2} X_{t 2}+\ldots+b_{n} X_{t n}+\varepsilon_{t}$.

A determination coefficient is used to assess the performance and accuracy of the proposed ANFIS and MLR. The resulting data are entered in Tab. V.

Various indicators can be used to assess model performance. MLR and ANFIS are compared using root mean squared error (RMSE) and coefficient of determination (R2). The results indicate that the generated neuro-fuzzy model is consistent and appropriate for sensitivity analysis in the US stock market. The determination coefficient shows a high value of 0.85 , compared to a low value of 0.26 multidimensional regression model. This suggests that artificial intelligence is a useful tool for leveraging the influence of input variables on the S\&P 500's stock index performance and can be used as an acceptable alternative to conventional regression models.

\section{DISCUSSION}

Based on the sensitivity analysis of the US stock market using artificial intelligence, we can say that the S\&P 500 is the most sensitive to the political stability index and the economic optimism index. Conversely, the control of corruption index and VIX have not shown a significant impact on the performance of the selected stock index. Babu and Kumar (2015) also analysed the impact of national sentiment represented by the VIX index and the Facebook GrossNational Happiness (FGNH) index on the NSE index, which is listed on the stock exchange in India. Sensitivity analyses conducted through a neuro-fuzzy approach confirmed that there is a significant relation between national sentiment and NSE return. As a part of the research carried out in this work, VIX is not of crucial importance to the S\&P 500 return. On the contrary, more sensitive indicators of the stock index performance were discovered in the research. One of them is the index of political stability. The more confidence investors have in a corrupt government and a stable political environment, the more they are willing to invest, and the performance of the index is growing.

Another similar work by Dhaoui and Khraief (2014) also used fuzzy logic to explain the intensity of trading and changes in business trends. Empirical findings of the sensitivity analysis show that pessimistic sentiment has a particularly significant impact on the development of the French financial market. In this paper, based on the ANFIS outputs, it can be clearly seen that, in addition to the political stability index, the index of economic optimism and favourable financial conditions of investors have a significant impact on the performance of the S\&P 500 index. According to the results of this work and previous research, it can be stated that the impact of pessimism exceeds the impact of optimism on the return of stock indices.

The author of the paper is not aware of other research based on artificial intelligence using investor sentiment and other psychological indicators of the stock index performance. However, it is necessary to point out the limitations of the used approach. These problems include the fact that there are no guarantees that the fuzzy system will remain stable. Moreover, it lacks the ability to learn and has no memory. The very determination of the appropriate membership function and fuzzy rules is very difficult in itself, since even after extensive testing it is not easy to say how many membership functions are necessary. Fuzzy system verification and validation requires extensive hardware testing in the loop, which may not be acceptable to all developers. The created model can be further refined to improve its performance, for example by validating the rules created by financial experts or analysts, or by selecting other input variables into the model and testing the created model on other stock markets or other stock indices. However, it should be noted that the created expert system is dedicated to one concrete problem only - the influence of investor sentiment represented by the VIX index, the influence of economic optimism, the index of political stability and the control of corruption on the adjusted return of the S\&P 500 index on the US stock market are analysed in concrete period. 


\section{CONCLUSION}

This paper deals with the use of artificial intelligence for sensitivity analysis in the US stock market. The created fuzzy model of the Takagi-Sugeno type contains four input variables and one output variable. The influence of investor sentiment represented by the VIX index, the influence of economic optimism, the index of political stability and the control of corruption on the adjusted return of the S\&P 500 index on the US stock market are analysed. Fuzzy rules are determined and tested using an adaptive neuro-fuzzy inference system. ANFIS generated a total of 81 rules, which are subsequently reduced by fuzzy clustering and seven rule clusters. The membership function is defined as a Gaussian function because it best matches the distribution of values derived from the analysed stock market. The main contribution of this paper is to perform sensitivity analysis using artificial intelligence methods on the US stock market using alternative psychological indicators. Paper offers some evidence indicating that investor sentiment plays a central role in explaining market trend changes. Empirical findings show that optimistic sentiment and political stability index have a particularly significant impact on the S\&P 500 performance. However, it should be noted that the created expert system is dedicated to one concrete problem only. Results indicate that incorporating psychological indicators in macroeconomic models leads to better supervision and control of the financial markets.

Acknowledgements

This paper was supported by project No. FP-J-19-5814 'The Use of Artificial Intelligence in Business III’ from the Internal Grant Agency at Brno University of Technology.

\section{REFERENCES}

ALAM, S. and NADEEM, M. 2015. Impact of macroeconomic factors on capital market of Pakistan: An empirical study. Journal of Business Strategies, 9(2): 47-58.

ALJAZAERLI, M. A., SIROP, R. and MOUSELLI, S. 2016. Corruption and Stock Market Development: New Evidence from GCC Countries. Verslas: Teorija ir Praktika, 17(2): 117-127.

BABU, A. S. and KUMAR, R. R. 2015. The Impact of Sentiments on Stock Market: A Fuzzy Approach. IUP Journal of Applied Finance, 21(2): 22-33.

BOLGORIAN, M. 2011. Corruption and stock market development: A quantitative approach. Physica A: Statistical Mechanics and its Applications, 390(23-24): 4514-4521.

BOYACIOGLU, M. A. and AVCI, D. 2010. An Adaptive Network-Based Fuzzy Inference System (ANFIS) for the prediction of stock market return: The case of the Istanbul Stock Exchange. Expert Systems with Applications, 37(12): 7908-7912.

CASTILLO, O., MELIN, P., KACPRZYK, J. and PEDRYCZ, W. 2007. Type-2 Fuzzy Logic: Theory and Applications. In: IEEE International Conference on Granular Computing (GRC). November, Fremont, CA, USA: IEEE, pp. 145-145.

CHANG, P. C., FAN, C. Y. and LIN, J. L. 2011. Trend discovery in financial time series data using a case based fuzzy decision tree. Expert Systems with Applications, 38(1): 6070-6080.

CHAU, F., DEESOMSAK, R. and WANG, J. 2014. Political uncertainty and stock market volatility in the Middle East and North African (MENA) countries. Journal of International Financial Markets, Institutions and Money, 28: 1-19.

DASZYNSKA-ZYGADLO, K., SZPULAK, A. and SZYSZKA, A. 2014. Investor sentiment, optimism and excess stock market returns. Evidence from emerging markets. Business and Economic Horizons, 10(4): 362-373.

DHAOUI, A. and KHRAIEF, N. 2014. Sensitivity of trading intensity to optimistic and pessimistic beliefs: Evidence from the French stock market. Arab Economic and Business Journal, 9(2): 115-132.

DOSTÁL, P. and KRULJACOVÁ, A. 2018. Evaluation of University Quality via Fuzzy Logic. In: Inovation Management and Education Excellence through Vision 2020. 15-16 November, Sevilla, Spain: IBIMA, pp. 1368-1375.

DOSTÁL, P. 2011. Advanced Decision Making in Business and Public Services. Brno: Academic Publishing House CERM.

DOSTÁL, P., RAIS, K. and SOJKA, Z. 2005. Advanced methods of managerial decision-making: concrete examples of using methods in practice [in Czech: Pokročilé metody manažerského rozhodování: konkrétní př́klady využití metod v praxi]. Praha: Grada. Expert (Grada).

ESFAHANIPOUR, A. and AGHAMIRI, W. 2010. Adapted Neuro-Fuzzy Inference System on indirect approach TSK fuzzy rule base for stock market analysis. Expert Systems with Applications, 37(7): 4742-4748. 
FERNANDES, N. and GONENC, H. 2016. Multinationals and cash holdings. Journal of Corporate Finance, 39: 139-154.

FINANCE YAHOO. 2019. S\&P 500 index. Yahoo Finance. [Online]. Available at: https://finance.yahoo. com/quote/\%5EGSPC?p=^GSPC [Accessed: 2019, April 13].

GARCÍA, F., GUIJARRO, F., OLIVER, J. and TAMOŠIUUNIENĖ, R. 2018. Hybrid Fuzzy Neural Network to Predict Price Direction in the German DAX-30 Index. Technological and Economic Development of Economy, 24(6): 2161-2178.

GHANI, U., BAJWA, I. and ASHFAQ, A. 2018. A Fuzzy Logic Based Intelligent System for Measuring Customer Loyalty and Decision Making. Symmetry, 10(12): 761.

HAMMER, M., JANDA, O. and ERTL, J. 2012. Selected Soft-Computing Methods in Power Oil Transformer Diagnostics - part 1. [in Czech: Využití vybraných soft-computingových metod v diagnostice výkonových olejových transformátorů - 1. Část]. Elektrorevue, 14(3): 33.

JEFFERSON, C., LIU, H. and COCEA, M. 2017. Fuzzy Approach for Sentiment Analysis. In: IEEE International Conference on Fuzzy Systems. 9. July. Naples, Italy: IEEE, pp. 1-6.

KLIGER, D. and KUDRYAVTSEV, A. 2013. Volatility expectations and the reaction to analyst recommendations. Journal of Economic Psychology, 37(C): 1-6.

KLIGER, D. and LEVY, O. 2003. Mood-induced variation in risk preferences. Journal of Economic Behaviour and Organization, 52: 573-584.

LAU, C. K. M., DEMIR, E. and BILGIN, M. H. 2013. Experience-based corporate corruption and stock market volatility: Evidence from emerging markets. Emerging Markets Review, 17: 1-13.

LIU, H. and COCEA, H. 2017. Fuzzy Rule Based Systems for Interpretable Sentiment Analysis. In: 2017 Ninth International Conference on Advanced Computational Intelligence (ICACI). 4-6 February, Doha, Qatar: IEEE, pp. 129-136.

LOEWENSTEIN, G. F., HSEE, C. K., WEBER, E. V. and WELCH, N. 2001. Risk as feelings. Psychological Bulletin, 127(2): 267-286.

MADHUSUDHANAN, S. and MOORTHI, M. 2018. Optimized fuzzy technique for enhancing sentiment analysis. Cluster Computing, (online only): doi.org/10.1007/s10586-017-1514-z.

NAMOURI, H., JAWADI, F., FTITI, Z. and HACHICHA, N. 2017. Threshold effect in the relationship between investor sentiment and stock market returns: a PSTR specification. Applied Economics, 50(5): 559-573.

NOVÁK, V. 2000. Basics of fuzzy modeling [in Czech: Základy fuzzy modelování]. Praha: BEN.

OMODERO, C. O. 2018. Corruption and stock market performance in Nigeria. Annals of Spiru Haret University. Economic Series, 18(4): 23-40.

PEROTTI, E. C. and OIJEN, P. V. 2001. Privatization, political risk and stock market development in emerging economies. J. Int. Money Finance, 20(1): 43-69.

RUAN, L. 2018. Research on Sustainable Development of the Stock Market Based on VIX Index. Sustainability, 10(11): 2-12.

SALLEH, M. N. M., TALPUR, N. and TALPUR, K. H. 2018. A Modified Neuro-Fuzzy System Using Metaheuristic Approaches for Data Classification. In: ACEVES-FERNANDEZ, M. A. Artificial Intelligence - Emerging Trends and Applications. IntechOpen.

SARWAR, G. 2014. U.S. stock market uncertainty and cross-market European stock returns. Journal of Multinational Financial Management, 28(C): 1-14.

SHRIVASTAVA, H. and SRIDHARAN, S. 2013. Conception of data preprocessing and partitioning procedure for machine learning algorithm. International Journal of Recent Advances in Engineering \& Technology, 1(3): 160-164.

TAKAGI, T. and SUGENO, M. 1985. Fuzzy identification of systems and its application to modeling and control. IEEE Trans. Syst. Man. Cybernet., 15(1): 116-132.

TALPUR, N., SALLEH, M. N. M. and HUSSAIN, K. 2017. An investigation of membership functions on performance of ANFIS for solving classification problems. In: IOP Conference Series: Materials Science and Engineering. 6-7 May. Melaka, Malaysia: IOP, pp. 1-7.

TASKIN, A. and KUMBASAR, T. 2015. An Open Source Matlab/Simulink Toolbox for Interval Type-2 Fuzzy Logic Systems. In: IEEE Symposium Series on Computational Intelligence. 7-10 December. Cape Town, South Africa: IEEE, pp. 1561-1568.

TRABELSI, M. A. 2017. Political uncertainty and behaviour of Tunisian stock market cycles: Structural unobserved components time series models. Research in International Business and Finance, 39: 206-214.

TUNG, K. T. and LE, M. H. 2017. An Application of Artificial Neural Networks and Fuzzy Logic on the Stock Price Prediction Problem. JOIV: International Journal on Informatics Visualization, 1(2): 40-49.

WANG, J. and WANG, J. 2015. Forecasting stock market indexes using principle component analysis and stochastic time effective neural networks. Neurocomputing, 156(1): 68-78. 
WORLD BANK. 2019. The Worldwide Governance Indicators (WGI) project. [Online]. Available at: http:// info.worldbank.org/governance/wgi/\#home [Accessed: 2019, April 13].

WRIGHT, W. F. and BOWER, G. H. 1992. Mood effects on subjective probability assessment. Organizational Behaviour and Human Decision Processes, 52(2): 276-291.

ZADEH, L. A. 1965. Fuzzy sets. Information and Control, 8(3): 338-353.

ZITTA, R. and PALATOVÁ, M. 2005. Methods for generating fuzzy rules from data [in Czech: Metody pro generování fuzzy pravidel z dat]. In: Konference Matlab 2005. Praha: Matlab, pp. 1-8. 